\title{
Emergence of a Powerful Connection Between Sensory and Cognitive Functions Across the Adult Life Span: A New Window to the Study of Cognitive Aging?
}

\author{
Paul B. Baltes and Ulman Lindenberger \\ Max Planck Institute for Human Development and Education
}

\begin{abstract}
Six hundred eighty seven individuals ages $25-103$ years were studied cross-sectionally to examine the relationship between measures of sensory functioning (visual and auditory acuity) and intelligence (14 cognitive tasks representing a 5-factor space of psychometric intelligence). As predicted, the average proportion of individual differences in intellectual functioning connected to sensory functioning increased from $11 \%$ in adulthood (25-69 years) to $31 \%$ in old age ( $70-103$ years). However, the link between fluid intellectual abilities and sensory functioning, albeit of different size, displayed a similarly high connection to age in both age groups. Several explanations are discussed, including a "common cause" hypothesis. In this vein, we argue that the increase in the age-associated link between sensory and intellectual functioning may reflect brain aging and that the search for explanations of cognitive aging phenomena would benefit from attending to factors that are shared between the 2 domains.
\end{abstract}

The role of sensory functions (such as vision and hearing) as antecedents, correlates, and consequents of intellectual functioning has not been at the center of research on the aging of intelligence, some exceptions notwithstanding (Granick, Kleban, \& Weiss, 1976; MacFarland, 1968; Nettelbeck \& Rabbitt, 1992; Stelmach \& Hömberg, 1993). To be sure, sensory functioning has been mentioned in general conceptual frameworks developed to index the realm of intellectual functioning (e.g., Carroll, 1993; Horn \& Hofer, 1992). Moreover, certain movements in the history of psychological theory, such as British empiricism or German elementarism (Hermstein \& Boring, 1965; Hilgard, 1987), have attended to the role of sensory input in the development and regulation of cognitive behavior. However, despite these lines of argument, in hundreds of studies on cognitive aging (for reviews, see Craik \& Salthouse, 1992; Salthouse, 1991b), sensory functioning and its relationship to

Data on the old-age sample (70-103 years) were collected as part of the Berlin Aging Study, which was sponsored by the Berlin-Brandenburg Academy of Sciences.

We express our gratitude to our many colleagues in this project, especially Markus Borchelt (Max Burger Hospital, Berlin) and Hans Scherer (professor and chairman of the Department of Otolaryngology, Free University of Berlin, Benjamin Franklin Medical College), who were significant partners in the decision to include sensory and sensorimotor measures in the assessment protocol of the Berlin Aging Study. In addition, we acknowledge helpful discussions with Chris Hertzog. Finally, we thank Annette Rentz, Anita Günther, Daniela Jopp, Karola Kersting, Gisela Schubert, Angelika Stöber, and Manfred Weilandt for assistance in data collection and project management regarding the younger sample ( $25-69$ years)

Correspondence concerning this article should be addressed to either Paul B. Baltes or Ulman Lindenberger, Center of Psychology and Human Development, Max Planck Institute for Human Development and Education, Lentzeallee 94, 14195 Berlin, Germany. complex intellectual functioning has rarely been part of the research agenda.

This relative disinterest in the role of sensory functioning in efforts to understand the aging of intelligence is probably due to at least three perspectives. First, there is the tradition of intelligence testing (Dixon \& Baltes, 1986; Sternberg \& Detterman, 1986), with its primary focus on complex and knowledgebased problem-solving tasks (however, see Vernon, 1987). Simple measures of visual and auditory acuity seemed ill-suited to capture the complexity of intelligence and related phenomena. Second, there is an age bias in research on intellectual development, with the vast majority of intelligence-related research being conducted with children and younger adults such as college students. Similarly, in cognitive aging research, samples rarely reach into advanced old age. If sensory systems operate at relatively high levels in most individuals during these earlier age periods, the likelihood of finding strong relations to other domains of functioning would seem to be reduced. Third, there possibly was a lack of knowledge among gerontological researchers interested in the study of intellectual functioning about the brain-based sources of age differences in visual and auditory acuity. Only more recently, cognitive aging researchers have begun to recognize more fully that age-related individual differences in visual and auditory functioning are not only peripheral phenomena but also reflect age-based changes in the central nervous system (cf. Fozard, 1990)

It would be dishonest to imply that the present line of work was primarily the outcome of hypothesis-guided research or of anticipatory insights into the potential role of sensory factors in cognitive aging. It is more accurate to state that, aside from an early interest in the topic by one of us (Schaie, Baltes, \& Strother 1964), the initial impetus was more due to the serendipities arising from interdisciplinary collaboration. In part, the data for the present study are taken from the Berlin Aging Study (BASE; Baltes, Mayer, Helmchen, \& Steinhagen-Thiessen, 
1993). Because of its interdisciplinary emphasis, researchers in BASE range widely across disciplines and include biological, medical, behavioral, and social scientists. Not surprisingly, therefore, the measurement scheme developed for BASE (which covered for each participant a total of 14 sessions of assessment) included measures of sensory functioning.

In our first report from BASE on the interface between sensory functioning and intelligence in old age (Lindenberger \& Baltes, 1994), the promise of interdisciplinary research materialized in a new finding. When considering the age range from 70 to 103 years, simple identification and threshold measures of visual and auditory acuity together accounted for $49 \%$ of the total and $93 \%$ of the age-related reliable variance in intellectual functioning. A large and fairly comprehensive battery of 14 tests of cognitive functioning (Lindenberger, Mayr, \& Kliegl, 1993) had been used to mark the intellectual ability domain. Thus, it was difficult to argue that this finding was specific to one type of cognitive task. Moreover, the findings presented in Lindenberger and Baltes (1994) were not of the significant-but-loweffect-size kind. On the contrary, the pattern of outcomes suggested that, in old age, measures of visual and auditory acuity were as good in predicting age-related individual differences in intellectual abilities as measures from the realm of intellectual functioning itself that are known to excel in this regard (e.g., measures of perceptual speed; cf. Salthouse, 1991a). In fact, the connection between sensory functioning and intelligence was so strong that the data were consistent with a structural model (Bentler, 1989) in which age differences in intelligence (as indexed by the common variance of perceptual speed, reasoning, memory, knowledge, and fluency) were completely mediated by differences in visual and auditory acuity.

In our first efforts at accounting for these findings (Baltes \& Lindenberger, 1995; Lindenberger \& Baltes, 1994) we identified three different but possibly interrelated interpretations: (a) the sensory deprivation hypothesis, (b) the hypothesis of an aginginduced increase in the cognitive load of sensory performance, and (c) the brain-related "common cause" hypothesis. The common cause explanation, which is at the focus of the present study, was based on the argument that correlations between measures of sensory and intellectual functioning may increase in old age because "both sets of measures are an expression of the physiological architecture ... of the [aging] brain", (Lindenberger \& Baltes, 1994, p. 339).

The common cause hypothesis was further supported by data analyses showing that the magnitude of the relation between sensory and cognitive functioning was largely invariant across types of cognitive tests, levels of sensory or cognitive performance, and absence versus presence of brain-related pathology (i.e., dementia). Moreover, an index of sensorimotor functioning-balance/gait-was found to display as high a connection to intellectual functioning as visual and auditory acuity. It should be noted, however, that the common cause interpretation of the age-based link between sensory and cognitive functioning is a third-variable hypothesis. Thus, in principle, the ensemble of common cause factors promoting the strong connection between the two domains of functioning may not only involve age-related changes in brain integrity, but also age-based changes in other bodily functions. Finally, we cannot exclude the possibility that age- or cohort-associated changes in experiential conditions, such as differences in life contexts and life events, also contribute to the connection.

The present study has two major goals: to replicate the original finding of a strong link between sensory functioning and intelligence in old age, and to explore differences in the magnitude of this link across the entire adult age span. The findings reported in Lindenberger and Baltes (1994) were based on the opening participant wave of BASE, which provided a random sample of 156 persons stratified by age and gender ranging from 70 to 103 years. The first objective of the present studyreplication-was achieved by extending that sample to the now available entire BASE sample, which consists of 516 persons, again stratified by age and gender and ranging from 70 to 103 years. The second objective-comparisons across a broader age span-was achieved by considering younger individuals. Specifically, we collected the same sensory and intellectual measures in a sample of younger adults $(N=171)$ ranging from 25 to 69 years.

\section{Method}

\section{Samples and Procedure}

Younger adult sample (25-69 years). Younger individuals ( $N=$ $171, M$ age $=48.2$ years, $S D=14.7,58 \%$ women) were recruited by a survey research institute to obtain a heterogeneous sample base and were tested individually at our laboratory in two sessions. In the first session, a general information questionnaire (modeled after the Intake Assessment of BASE; cf. Baltes et al., 1993) as well as measures related to vision and hearing were given. The battery of cognitive tests was administered in the second session. Procedures for sensory and cognitive assessments ( see below) were identical to those used in BASE and have been reported in more detail elsewhere (Baltes \& Lindenberger, 1995; Lindenberger et al., 1993; Steinhagen-Thiessen \& Borchelt, 1993).

BASE sample (70-103 years). Older individuals ( $N=516, M$ age $=84.9$ years, $S D=8.66$ ) represent the total sample of BASE (cf. Baltes et al., 1993). The sample is stratified by gender and six age brackets (70$74,75-79,80-84,85-89,90-94$, and $95+$ years ), with 43 individuals in each of the resulting 12 design cells. Measures of distance visual acuity were given at the beginning, the battery of cognitive tests in the middle, and measures of hearing and close visual acuity toward the end of the 14-session multidisciplinary assessment protocol (for details, see Lindenberger \& Baites, 1994).

Composite sample (25-101 years). Some of the results reported below are based on regression analyses that cover an age range from 25 to over 100 years. For these analyses, the new younger sample was combined with a subsample from BASE rather than with all 516 participants to ensure that all levels of the independent variable (i.e., chronological age) were given about equal weight in the regression equations. Specifically, 12 individuals were drawn at random from each of the 12 Gender (2) $\times$ Age Group (6) design cells of BASE, with the constraints that they had not received a clinical diagnosis of dementia according to criteria from the revised third edition of the Diagnostic and Statistical Manual of Mental Disorders (DSM-III-R; American Psychiatric Association, 1987) and that they were not part of the sample reported in Lindenberger and Baltes (1994). The resulting sample of 144 individuals was combined with the new younger sample to yield a composite sample $(N=315)$ that spanned an age range from 25 to 101 years ( $M$ age = 64.9 years, $S D=22.0$ )

\section{Measures}

Visual acuity. Visual acuity was measured in Snellen decimal units at two different distances using two different standard reading tables 
containing digits and letters (Geigy, 1977). Distance visual acuity was assessed binocularly using a reading table presented at a standard distance of $2.5 \mathrm{~m}$ to the participant. Close visual acuity was measured separately for the left and the right eye using a reading table presented at reading distance. All three measurements were taken without and with the best optical correction (i.e., glasses) available to the participant. Ninety two percent of the participants in the BASE sample and $74 \%$ of the participants in the younger sample had at least one pair of glasses. The analyses reported in this article are based on the better values, which in most cases referred to corrected vision. The decision to use corrected rather than uncorrected vision is in line with our earlier work (Baltes \& Lindenberger, 1995; Lindenberger \& Baltes, 1994). It also provides for a better test of the common cause hypothesis because corrective devices should filter out, to a certain degree, peripheral variance (e.g., variance due to individual differences in the refractory properties of the lens), thereby allowing for a more direct assessment of the portion of sensory loss that is central-neuronal in nature.

Auditory acuity. Measures related to auditory acuity were assessed with a Bosch ST-20-1 pure tone audiometer using headphones. Thresholds were measured separately for the right and left ears at eight different frequencies. Sixteen percent of the individuals in the BASE sample and none of the individuals in the younger sample had hearing aids. For technical reasons, thresholds were assessed without hearing aids only. Testing started with the better ear; for participants who did not know which ear was their better one, testing started with the right ear. Within ears, frequencies were tested in the following order: $1.00,2.00,3.00$, $4.00,6.00,8.00,0.50$, and $0.25 \mathrm{kHz}$.

Cognitive test battery. A total of 14 tests was administered measuring five different intellectual abilities: perceptual speed (Digit Letter Test, Digit Symbol Substitution, Identical Pictures), reasoning (Figural Analogies, Letter Series, Practical Problems), memory (Activity Recall, Memory for Text, Paired Assocíates), knowledge (Practical Knowledge, Spot-a-Word, Vocabulary), and fluency (Animals, Letter 'S'"). Perceptual speed, reasoning, and memory represent the broad fluid domain (Horn, 1982) or the relatively knowledge-free "mechanics" of cognition (Baltes, 1987, 1993). In contrast, knowledge and fluency represent the broad crystallized domain of cognitive functioning, or the knowledgesaturated "pragmatics." Stimulus presentation and data collection were supported by a Macintosh SE30 personal computer equipped with a MicroTouch Systems touch-sensitive screen. A detailed description of the tests and their psychometric and structural properties can be found elsewhere (Lindenberger et al., 1993). The 14 measures have satisfactory internal consistencies, high interrater reliabilities, and substantial loadings on their latent factors (Lindenberger et al., 1993). Using exploratory and confirmatory factor-analytic techniques (cf. Hertzog, 1990; McArdle \& Nesselroade, 1994), structural differentiation into the five expected latent ability factors could be demonstrated for all three samples reported in this study.

\section{Results}

\section{Overview}

Results are reported in three sections. First, we report crosssectional age gradients (Baltes, 1968; Schaie, 1965) of the five intellectual abilities in the composite life span sample (age range $=25-101$ years ) and examine the extent to which age gradients in intellectual abilities are attenuated after regressing intellectual abilities on vision, hearing, or both. Second, we investigate whether vision and hearing are more strongly related to individual differences in intellectual abilities in the old sample than in the younger sample. Third, we examine whether intercorrelations within the sensory and cognitive domains were higher in the older sample. To minimize the complexity of statistical procedures, the relevant variables of sensory and cognitive functioning are based on composites of their standardized indicators and were scaled in a $T$-score metric (i.e., $M=50, S D=10$ ). Thus, measures are not corrected for unreliability through the use of structural modeling techniques, as we had done in previous work (Lindenberger \& Baltes, 1994). In analyses not reported here, equivalent results were obtained using structural modeling techniques.

\section{Cross-Sectional Age Gradients in Intellectual Abilities From Age 25 to 101 Years: The Connection to Vision and Hearing}

Figure 1 and the top rows of Table 1 display the relation of vision, hearing, and intellectual abilities to the linear and quadratic trends of age.' The table also contains an 'intellectual ability composite" computed as the unit-weighted average over the five intellectual abilities to represent the centroid of the intellectual ability factor space as assessed with our battery. On average, the age gradients of vision and hearing were more negative than the age gradients of the three fluid-mechanical intellectual abilities of perceptual speed, reasoning, and memory $(z=3.76, p<.01)$, which in turn-as predicted by life span theories of psychometric intelligence (Baltes, 1993; Horn \& Hofer, 1992; Schaie, 1965) -had more negative gradients than knowledge and fluency, the two crystallized pragmatic abilities $(z=8.77, p<.01){ }^{2}$ Post hoc comparisons revealed the following order in the magnitude of negative age relations $(p<.01)$ : hearing $>$ perceptual speed $>$ (reasoning $=$ vision $=$ memory $)$ $>$ (knowledge $=$ fluency $)$.

Next, we examined whether age-related individual differences in the two domains were relatively independent of each other, or whether a large portion of the age-related variance in the two domains was shared. The middle rows of Table 1 provide a clear answer to this question. Averaged across the five intellectual abilities, controlling for vision was associated with a 3.9fold reduction of age-related individual differences in cognitive functioning. Controlling for hearing led to a 8.8-fold reduction, and controlling for both vision and hearing to a 18.5 -fold reduction of age differences in cognitive functioning. In fact, most linear and quadratic age trends in intellectual abilities were no longer significantly different from zero $(p<.01)$ after controlling for either hearing alone or vision and hearing. ${ }^{3}$

\footnotetext{
' The quadratic age trend was computed by regressing age squared on age and saving the residuals (i.e., the quadratic component that is orthogonal to the linear component of age).

${ }^{2}$ Within-sample differences in age gradients were tested for significance using the formulae described in Meng, Rosenthal, and Rubin (1992). Correlations of intellectual abilities with both linear and quadratic age trends were taken into consideration when testing for differences in age gradients [i.e., $R$ (age $)=\left(r_{\text {age linear }}^{2}+r_{\text {age quadraicic }}^{2}\right)^{1 / 2}$ ].

${ }^{3}$ When regressing the five intellectual abilities on vision and hearing in the composite sample (i.e., $N=315$, age range $=25-101$ years), the Vision $\times$ Hearing interaction accounted for about an additional $1 \%$ of predicted variance $(p<.01)$ in all five intellectual abilities. For this reason, the interaction term was included in regression equations with the composite sample when both vision and hearing served as predictor variables.
} 

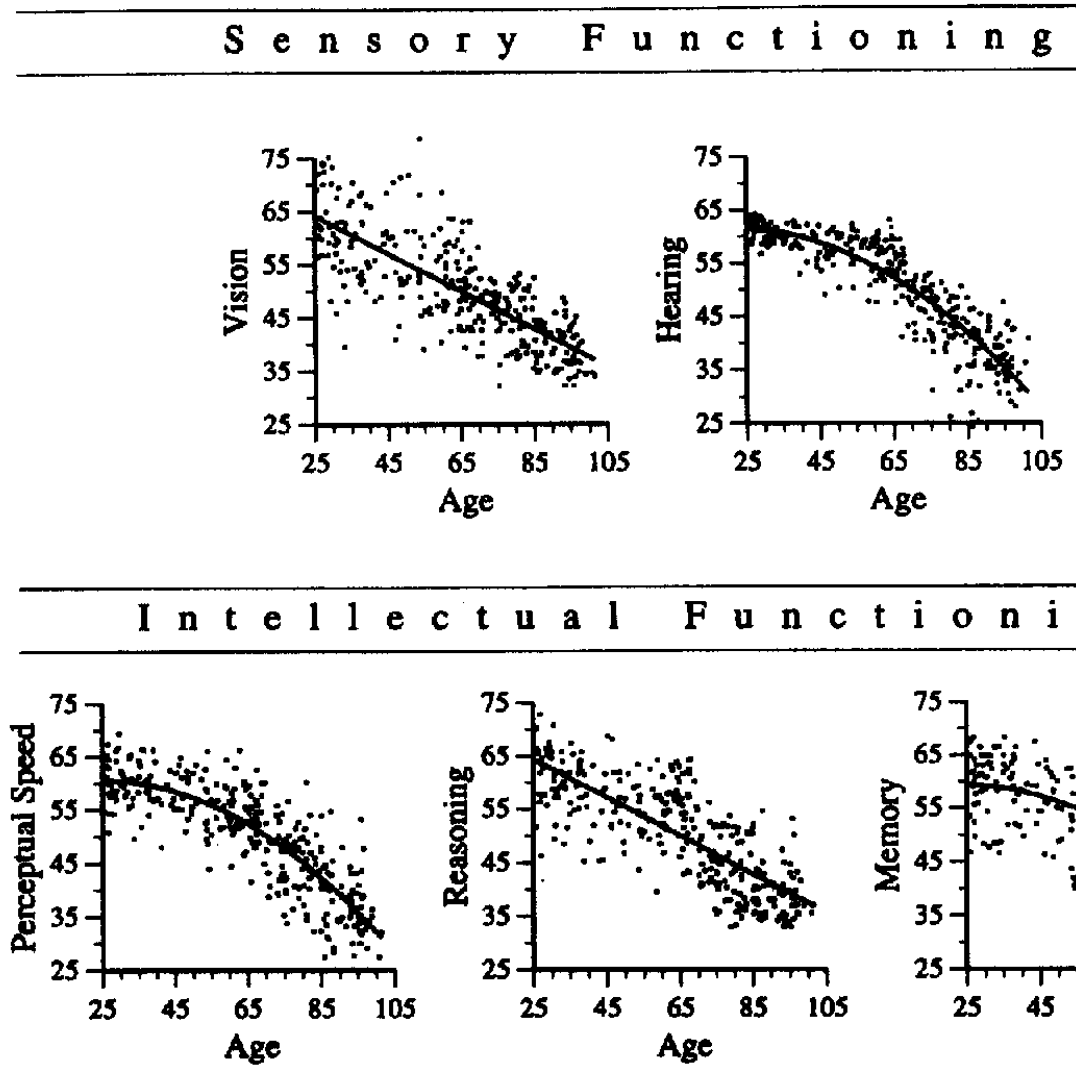

c t

F
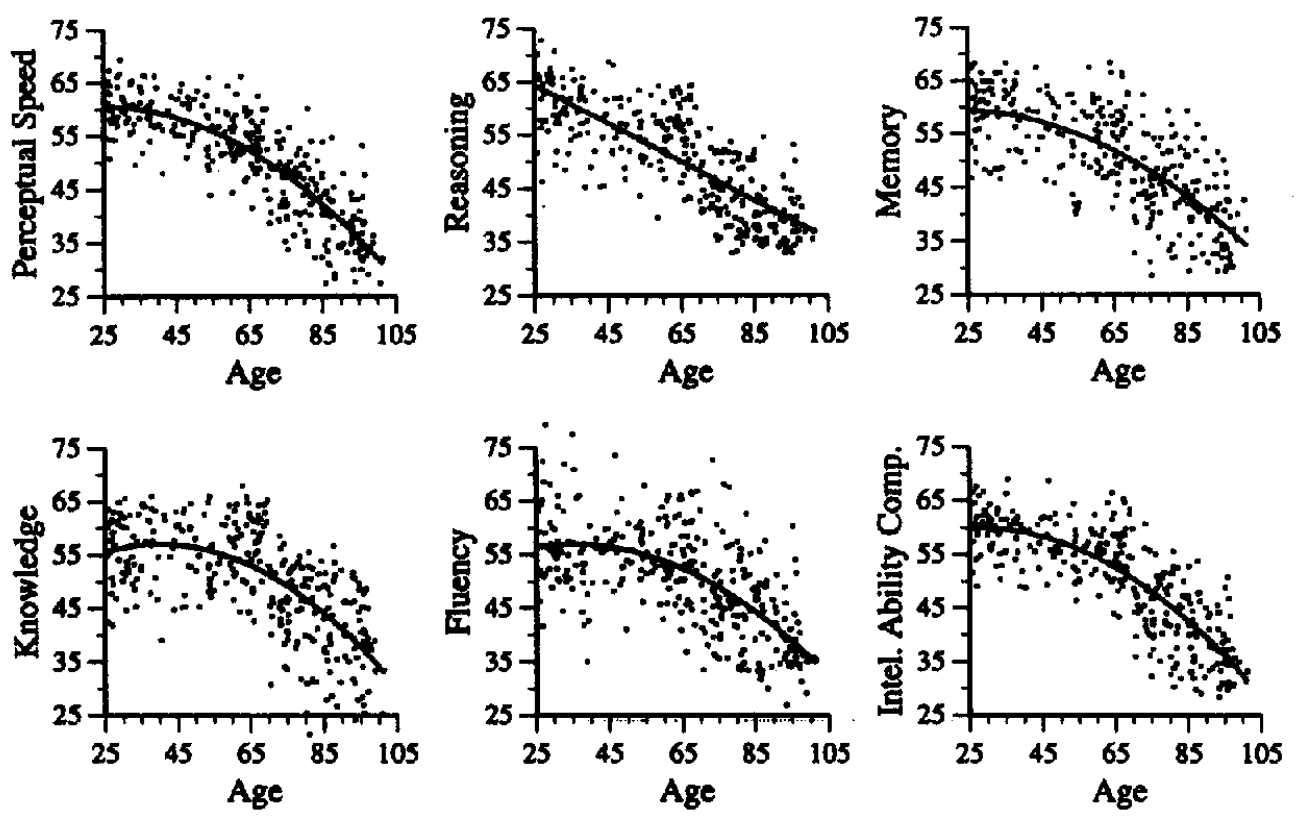

Figure 1. Cross-sectional age gradients for vision, hearing, five intellectual abilities, and the intellectual ability composite $(N=315$, age range $=25-101$ years $)$. Linear and quadratic age trends are reported in the top rows of Table 1 . With respect to vision and reasoning, quadratic age trends did not differ significantly from zero $(p>.01)$. Intel. Ability Comp. = intellectual ability composite.

The bottom rows of Table 1 also show that controlling for individual differences in vision and hearing was about as effective in reducing the age-related variance in the remaining four intellectual abilities as controlling for perceptual speed, the intellectual ability with the strongest negative relationship to age (cf. Lindenberger \& Baltes, 1994; Lindenberger et al., 1993). This is important because perceptual speed has been widely discussed as a primary mediator of negative age differences in adult cognition (Salthouse, 1991b).

Table 2 displays the link between sensory and cognitive functioning before and after regressing the intellectual abilities on linear and quadratic trends of age. On average, controlling for age was associated with a 14.1 -fold reduction in the amount of variance in intellectual functioning associated with vision and hearing.

Finally, Table 3 reports the unique and shared variance components of the main effects of vision, hearing, and age as predictors of the intellectual ability composite (commonality analysis; cf. Hertzog, 1989). Vision, hearing, or both accounted for $67.7 \%$ of the total, $94.7 \%$ of the age-related, but only $12.6 \%$ of the total age-independent variance in the intellectual ability composite. The latter figure (i.e., $12.6 \%$ ) was computed as the age-independent variance predicted by vision and hearing over the total amount of age-independent variance-that is, 100 * $\{(2.5+1.1+0.5) /[100-(3.8+3.2+12.9+47.5)]\}$. Moreover, $66.4 \%$ of the predicted variance was shared among all 
Table 1

Correlations of Intellectual Abilities With Simple (i.e., Linear) and Quadratic Age Trends Before and After Controlling for Individual Differences in Vision, Hearing, Vision and Hearing, or Perceptual Speed

\begin{tabular}{|c|c|c|c|c|c|c|c|c|}
\hline \multirow[b]{2}{*}{ Correlation } & \multicolumn{2}{|c|}{$\begin{array}{l}\text { Sensory } \\
\text { functioning }\end{array}$} & \multicolumn{6}{|c|}{ Cognitive functioning (intelligence) } \\
\hline & Vision & Hearing & $\begin{array}{l}\text { Perceptual } \\
\text { speed }\end{array}$ & Reasoning & Memory & Knowledge & Fluency & Composite \\
\hline \multicolumn{9}{|l|}{ Zero-order age correlations } \\
\hline Linear age & -.78 & -.87 & -.82 & -.79 & -.71 & -.61 & -.60 & -.79 \\
\hline Quadratic age & -.05 & -.21 & -.22 & -.12 & -.17 & -.28 & -.22 & -.23 \\
\hline Variance predicted $(\%)$ & 60.6 & 79.2 & 71.5 & 64.1 & 52.8 & 45.0 & 40.4 & 67.4 \\
\hline \multicolumn{9}{|l|}{$\begin{array}{l}\text { Age correlations of residuals } \\
\text { controlled for individual } \\
\text { differences in vision }\end{array}$} \\
\hline Linear age & - & -.44 & -.36 & -.34 & -.29 & -.18 & -.16 & -.32 \\
\hline Quadratic age & - & -.25 & -.27 & -.12 & -.18 & -.32 & -.24 & -.28 \\
\hline Variance predicted $(\%)$ & - & 27.7 & 19.0 & 15.5 & 15.8 & 12.1 & 8.5 & 16.7 \\
\hline \multicolumn{9}{|l|}{$\begin{array}{l}\text { Age correlations of residuals } \\
\text { controlled for individual } \\
\text { differences in hearing }\end{array}$} \\
\hline Linear age & -.22 & - & -.21 & -.20 & -.13 & -.06 & -.10 & -.17 \\
\hline Quadratic age & .15 & - & -.09 & -.06 & -.04 & -.19 & -.12 & -.10 \\
\hline Variance predicted $(\%)$ & 9.5 & - & 9.1 & 7.2 & 4.6 & 4.4 & 5.8 & 7.0 \\
\hline \multicolumn{9}{|c|}{$\begin{array}{l}\text { Age correlations of residuals } \\
\text { controlled for individual } \\
\text { differences in vision and hearing }\end{array}$} \\
\hline Linear age & - & - & -.16 &.- .13 & -.10 & -.02 & -.05 & -.12 \\
\hline Quadratic age & - & - & -.05 & .01 & .01 & -.13 & -.10 & -.07 \\
\hline Variance predicted $(\%)$ & - & - & 4.6 & 3.4 & 2.7 & 1.8 & 2.3 & 3.0 \\
\hline \multicolumn{9}{|c|}{$\begin{array}{l}\text { Age correlations of residuals } \\
\text { controlled for individual } \\
\text { differences in perceptual speed }\end{array}$} \\
\hline Linear age & -.26 & -.36 & - & -.21 & -.10 & .01 & .01 & - \\
\hline Quadratic age & .17 & -.05 & - & .11 & .00 & -.18 & -.09 & - \\
\hline Variance predicted $(\%)$ & 9.0 & 16.5 & - & 4.3 & 1.2 & 1.5 & 0.4 & - \\
\hline
\end{tabular}

Note. $\quad N=315$, age range $=25-101$ years. The quadratic component of age was computed by regressing age squared on age (i.e., linear age). Residual age correlations were computed by correlating residuals controlled for individual differences in the corresponding variable(s) with linear and quadratic trends of age. Portions of variance associated with linear and quadratic age trends were obtained by means of hierarchical regressions and may differ somewhat from the sum of squared linear and quadratic age trends due to collinearities among predictors. Coefficients not significantly different from zero at the .01 level are in boldface. Dashes refer to values that were not computed because of complete or partial identity between the dependent and independent variables.

three predictors, and a substantial portion of the total variance, $12.9 \%$, was shared between age and hearing only.

The results reported in this section show a powerful intersystemic connection. Age gradients in five different intellectual abilities were extremely well predicted by individual differences in vision and hearing. An average of $92.9 \%$ of the predictive variance in vision and hearing was shared with age. In the next section, we investigate whether this link between sensory and intellectual functioning increases from adulthood to old age.

\section{Age Differences in the Link Between Sensory and Intellectual Functioning: 25-69 Years Versus 70-103 Years}

Figure 2 shows the amount of total variance accounted for by vision and hearing in the five different intellectual abilities and the intellectual ability composite. ${ }^{4}$ Averaged over the five different intellectual abilities, vision and hearing predicted about $11 \%$ of the total variance in the younger sample $(N=171$, age range $=25-69$ years $)$, but about $31 \%$ of the total variance in the old sample ( $N=516$, age range $=70-103$ years $)$. Except for reasoning $(z=1.94, p=.026)$, the amount of variance predicted by vision and hearing was significantly larger in the old-age sample (perceptual speed: $z=2.98$; memory: $z=3.67$; knowledge: $z=4.93$; fluency: $z=3.28$; intellectual ability composite: $z=3.46$; for all $z$ values, $p$ s $<.01$ ). Within the old-age sample, vision and hearing predicted more variance in perceptual speed than in the other four intellectual abilities ( $z$ $=5.57$ ). An examination of the joint and unique contributions of vision and hearing suggested that this surplus covariance was due, for the most part, to individual differences in vision, and possibly reflects the visual nature of the perceptual speed measures (see also Lindenberger \& Baltes, 1994).

The data summarized in Figure 2 lend strong support to our

\footnotetext{
${ }^{4}$ In both samples, quadratic age trends did not differ from zero (all $p \mathrm{~s}$ $>.01$ ), and Vision $\times$ Hearing interactions did not explain a significant additional amount of variance in any of the variables from the intellectual domain. Therefore, results reported in this section are based on linear age trends and on main effects of vision and hearing only.
} 
Table 2

Correlations of Intellectual Abilities With Vision and Hearing Before and After Controlling for Individual Differences in Linear and Quadratic Age

\begin{tabular}{lcccccc}
\hline \multicolumn{1}{c}{ Correlations } & $\begin{array}{c}\text { Perceptual } \\
\text { speed }\end{array}$ & Reasoning & Memory & Knowledge & Fluency & Composite \\
\hline $\begin{array}{l}\text { Zero-order correlations } \\
\quad \text { Vision }\end{array}$ & .74 & .71 & .62 & .60 & .60 & .73 \\
$\quad$ Hearing & .80 & .76 & .71 & .66 & .59 & .79 \\
$\quad \begin{array}{l}\text { Variance predicted (\%) } \\
\text { Correlations of residuals }\end{array}$ & 70.5 & 63.9 & 53.3 & 48.7 & 42.3 & 68.9 \\
$\quad \begin{array}{l}\text { controlled for linear } \\
\text { and quadratic age trends }\end{array}$ & & & & & & \\
$\quad$ Vision & .18 & .15 & .09 & .15 & .16 & .19 \\
Hearing & .08 & .09 & .09 & .10 & .04 & .10 \\
$\quad$ Variance predicted $(\%)$ & 3.6 & 3.2 & 3.2 & 5.4 & 4.3 & 4.5 \\
\hline
\end{tabular}

Note. $\quad N=315$, age range $=25-101$ years. Residual correlations were computed by correlating residuals controlled for individual differences in linear and quadratic age trends with vision or hearing. Portions of variance associated with vision and hearing were obtained by means of hierarchical regressions and differ from the sum of squared vision and hearing correlations because of collinearities among the two predictors. Coefficients not significantly different from zero at the .01 level are in boldface.

expectation (Lindenberger \& Baltes, 1994) that vision and hearing are more closely related to intelligence in old age than during earlier periods of the adult life span. From the perspective of the common cause hypothesis, this age-associated increase in the link between sensory functioning and intelligence is induced by an age-based acceleration of changes in the central nervous system that affect both sensory and cognitive systems of functioning.

To further explore this idea, we examined whether the proportion of the predictive variance in vision and hearing that is shared with age would differ between the two age groups. As can be seen in Figure 3, shared variance proportions were high in both age groups for the three fluid abilities representing the mechanics of cognition, with no evidence for an increase between age groups. In contrast, values were considerably lower in the younger age group than in the old-age sample for knowledge and fuency, the two crystallized abilities representing the pragmatics of cognition. ${ }^{5}$ This is not surprising because these

Table 3

Predicting Interindividual Differences in the Intellectual Ability Composite: Unique and Shared Variance Components of Age, Vision, and Hearing

\begin{tabular}{lc}
\multicolumn{1}{c}{ Component } & Variance explained (\%) \\
\hline Unique age & 3.8 \\
Unique vision & 2.5 \\
Unique hearing & 1.1 \\
Shared age, vision & 3.2 \\
Shared age, hearing & 12.9 \\
Shared vision, hearing & 0.5 \\
Shared age, vision, hearing & 47.5 \\
Total variance explained & 71.5 \\
\hline
\end{tabular}

Note. $N=317$, age range $=25-101$ years. Variance components are based on unweighted composites and were computed by regressing the intellectual ability composite on all possible combinations of the main effects of age (linear and quadratic), vision, and hearing. two abilities showed no significant negative relation to age in the age range between 25 and 69 years (knowledge: $r=.06$, $n s$; fluency: $r=-13, n s$ ). The finding is also theoretically meaningful as life span theories of intelligence (Baltes, 1993; Horn \& Hofer, 1992) postulate that, during adulthood, crystallized pragmatic abilities primarily reflect cultural-experiential rather than biological factors.

The high degree of predictive overlap between age and sensory functioning for fluid abilities in both age groups is consistent with the notion that mechanisms associated with chronological age are the driving force behind the sensory-cognitive link across the entire age range considered in this study. From this perspective, the increase in the strength of the connection between sensory and intellectual functioning with advancing age is due to an acceleration of negative age-based changes in both domains during the last decades of the life span.

\section{Age Differences in Magnitude of Correlations Within Cognitive and Sensory Domains}

According to our common cause hypothesis, mechanisms related to brain aging function as a general and increasingly severe "common" constraint for many different functional systems of the brain. A corollary prediction emanating from this common causes-based view is that relations within sensory and cognitive domains should also increase with advancing age, reflecting the increasing importance of this general set of constraints ( $\mathrm{cf}$. the dedifferentiation hypothesis of old-age intelligence; Reinert, 1970). Thus, because of the increasing role of a common cause, not only the across-domain connections, but also the withindomain correlations should be higher in the old sample (70103 years) than in the younger sample (25-69 years).

The relevant correlations for the younger and the old-age

\footnotetext{
${ }^{5}$ A statistical test for across-sample differences in variance proportions is difficult to obtain because they represent different amounts of total variance.
} 


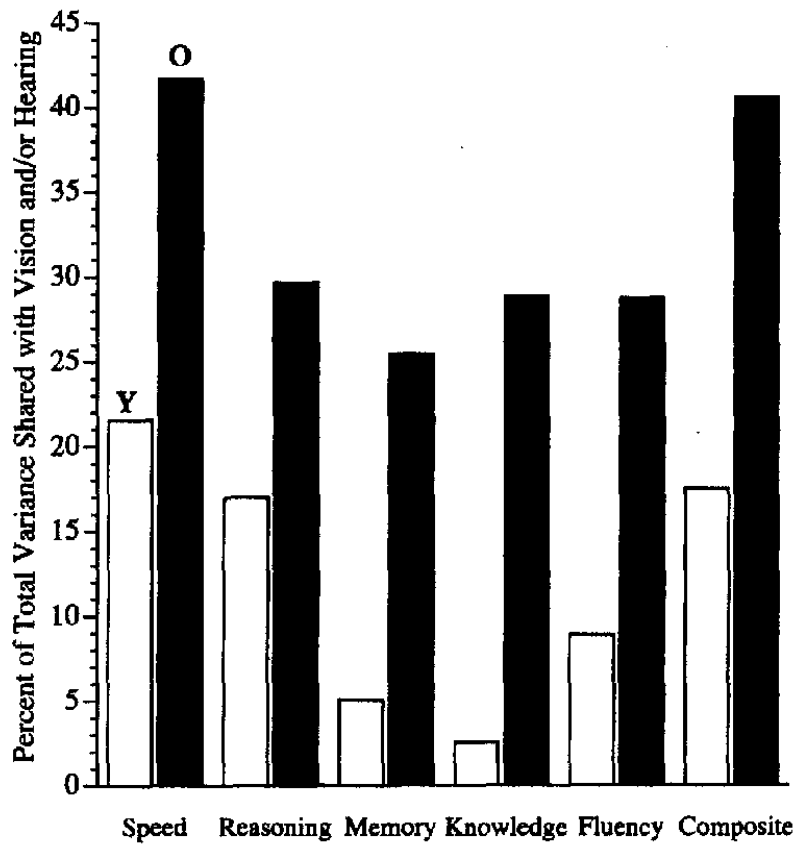

Figure 2. Amount of total variance in intellectual functioning accounted for by vision and hearing in two age groups. Bars represent the amount of total variance predicted by the main effects of vision and hearing. Light bars refer to the younger $(Y)$ sample $(N=171$, age range $=25-69$ years $)$, and dark bars to the old-age $(O)$ sample $(N=$ 516, age range $=70-103$ years $)$. Except for reasoning, differences in predicted variance were significant at the .01 level.

sample are reported in Table 4 . The median correlation among the five intellectual abilities was $r=.38$ in the younger and $r$ $=.71$ in the old sample; the difference was statistically significant $(z=5.48, p<.01)$. For the correlation between vision and hearing, the difference was in the expected direction but statistically not reliable (younger sample: $r=.36$; old sample: $r=.45 ; z=1.17, p>.05)$.

\section{Summary}

Results can be summarized in five points. First, simple measures of vision and hearing showed negative cross-sectional age gradients of substantial magnitude over an age range of 25 to 101 years (Figure 1). Second, negative age gradients in intellectual abilities were extremely well predicted by individual differences in vision and hearing (Table 1 ). Third, as revealed by age group comparisons (i.e., $25-69$ vs. $70-103$ years), the link between sensory and intellectual functioning increased substantially from adulthood to old age (Figure 2). Fourth, with respect to fluid intellectual abilities or the mechanics of cognition, a high degree of age-relatedness of the link between sensory and intellectual functioning was observed in both age groups (Figure 3 ). Finally, intercorrelations within the cognitive domain were higher in the old-age than in the younger group (Table 3 ).

Before discussing these results, a methodological issue related to the interpretation of group differences in prediction needs to be addressed. If the variances of the relevant measures were systematically smaller in one of the two groups, such differences would tend to favor smaller covariances and, as a consequence, smaller regression coefficients in the less variable group (the so-called restriction-of-range problem). Compared with the oldage sample, the younger sample was actually more heterogeneous in three variables: age (Cochran's $C=.74$ ), vision ( $C$ $=.71$ ), and fluency ( $C=.57$; for all differences, $N_{1}=171, N_{2}$ $=516, p<.01)$. It was less heterogeneous in four variables: hearing (probably because hearing was measured in decibel units; $C=.67)$, perceptual speed $(C=.70)$, memory $(C=$ .60 ), and knowledge ( $C=.66$; for all differences, $N_{1}=171$, $N_{2}=516, p<.01$ ). No significant group differences in heterogeneity were found for reasoning (Cochran's $C=.56, p>$ $.01)$. Note also that the median correlation between vision and the five intellectual abilities was higher in the old-age sample, despite the fact that vision was more variable in the younger group ( $r=.29$ vs. $r=.49 ; z=2.57, p<.01$ ). On the basis of these analyses, it is unlikely that the observed age-group differences in the strength of the connection between sensory and intellectual functioning were largely due to statistical differences in sample variability.

\section{Discussion}

The main objective of this report is to present a new finding concerning the magnitude of the relationship between sensory

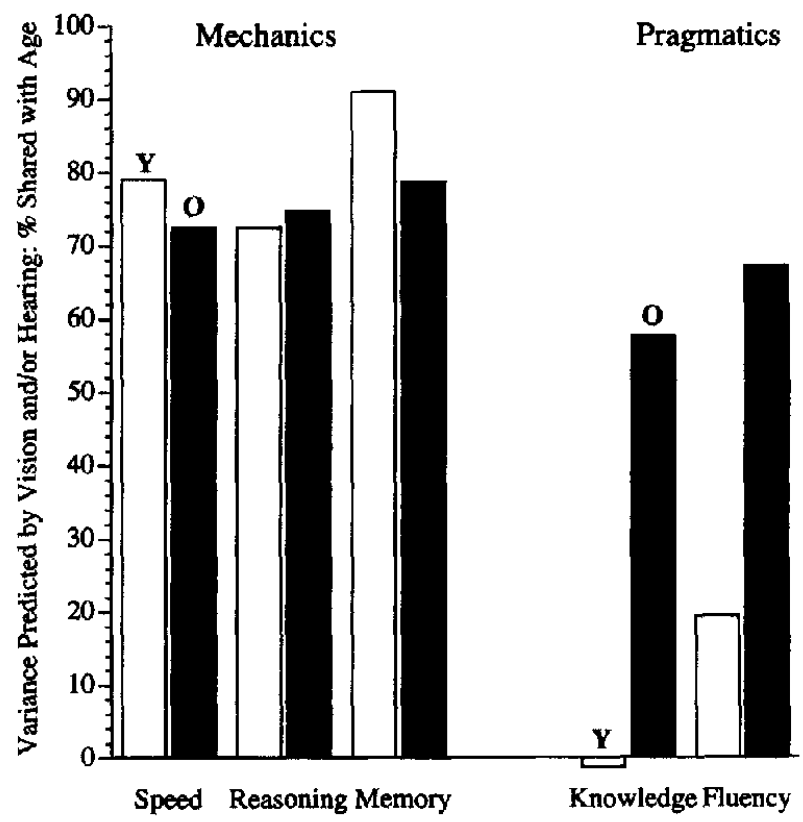

Figure 3. The proportion of variance in five intellectual abilities predicted by vision and hearing that is shared with age. Bars indicate how much of the variance predicted by vision and hearing is also predicted by age. The $100 \%$ reference points refer to the total amount of variance predicted by vision and hearing. For the mechanics of intelligence, the results show that the age-relatedness of the link between sensory and cognitive functioning is of equal magnitude in the two samples. Light bars refer to the younger (Y) sample $(N=171$, age range $=25-69$ years), and dark bars to the old-age (O) sample $(N=516$, age range $=70-103$ years . 
Table 4

Intercorrelations Among Vision, Hearing, and the Five Intellectual Abilities in the Younger (25-69 Years) and the Old (70-103 Years) Samples

\begin{tabular}{|c|c|c|c|c|c|c|c|c|}
\hline Item & 1 & 2 & 3 & 4 & 5 & 6 & 7 & 8 \\
\hline 1. Vision & - & .45 & .59 & .50 & .43 & .49 & .47 & -.59 \\
\hline 2. Hearing & .36 & - & .50 & .42 & .42 & .42 & .44 & -.57 \\
\hline 3. Perceptual speed & .43 & .32 & - & .72 & .71 & .71 & .73 & -.59 \\
\hline 4. Reasoning & .39 & .26 & .42 & - & .64 & .70 & .63 & -.51 \\
\hline 5. Memory & .20 & .17 & .42 & 40 & - & .66 & .70 & -.49 \\
\hline 6. Knowledge & .14 & -.02 & .22 & .42 & .42 & - & .70 & -.41 \\
\hline 7. Fluency & .29 & .05 & .36 & .30 & .25 & .33 & - & -.46 \\
\hline 8. Age & -.51 & -.63 & -.49 & -.41 & -.30 & .05 & -.13 & - \\
\hline
\end{tabular}

Note. Correlations for the old sample $(N=516, M=84.9$ years, range $=70-103$ years $)$ are shown above the main diagonal, and correlations for the younger sample $(N=171, M=48.2$ years, range $=25-69$ years) are shown below the main diagonal. Coefficients not significantly different from zero at the .01 level are in boldface.

systems and intelligence in old age. We submit that this new finding deserves serious consideration in adult-developmental and gerontological research on the aging mind. At the same time, we are aware that the work presented here needs to be complemented by other strategies of data collection such as cohort longitudinal methods to examine individual trends and possible cohort effects (Hultsch et al., 1992; Magnusson, Bergman, Rudinger, \& Törestad, 1991; Nesselroade \& Baltes, 1979; Schaie, 1995), more fine-grained and comprehensive methods of sensory assessment (Corso, 1987; Fozard, 1990), and componential analyses of the relevant cognitive and sensory tasks.

As is often true for novel findings obtained serendipitously in interdisciplinary research, the finding of a strong age-based and aging-induced link between sensory and cognitive functioning poses more questions than answers. Besides brain integrity, additional bodily and experiential factors may be involved (e.g., Welford, 1984; cf. Anstey, Lord, \& Williams, 1997). However, the magnitude of the intersystemic connection observed and the putative clarity of the data are promising and lend further support to the findings reported in Lindenberger and Baltes (1994). First, the replication part of the study showed that the results from the opening wave of BASE can be generalized to a larger sample. Second, the extension to younger age levels resulted in the predicted outcome: The relationship between sensory and cognitive functioning was much lower between 25 and 69 than between 70 and 103 years of age. At the same time, vision and hearing were excellent predictors of age differences in intellectual functioning across the entire age range represented in this study. This pattern of findings suggests that the mechanisms underlying the connection between sensory and cognitive functioning are similar across the entire adult life span, but that their expression is amplified in old and very old age.

In our previous work (Baltes \& Lindenberger, 1995; Lindenberger \& Baltes, 1994), we offered three hypotheses to explain the strong age-based connection between sensory and intellectual functioning: the sensory deprivation hypothesis, the aging-induced cognitive load hypothesis of sensory performance, and the common cause hypothesis. Currently, we favor the common cause hypothesis, according to which negative age differences in sensory and cognitive domains are the outcome of a third common factor or ensemble of factors, namely, the integrity of brain structure and function and its aging-induced changes. In contrast, the sensory deprivation hypothesis states that sensory functioning is closely related to intellectual functioning because protracted sensory underload and degradation of sensory input interfere with cognitive efficacy and, in the long run, reduce the likelihood of productive cognitive engagements. Further, the aging-induced cognitive load hypothesis would submit that seemingly "simple" sensory tasks increase in cognitive complexity and demands as participants reach old age.

The present results do not allow for a conclusive distinction among the three hypotheses and do not allow for the specification of underlying mechanisms. However, we believe that there is some further empirical support for the notion of a common cause. First, as reported elsewhere (Lindenberger \& Baltes, 1994 , p. 347), another measure of sensorimotor functioning, balance/gait, was found to show as high a connection to intellectual functioning in old age as measures of visual and auditory acuity. Second, the strong connection between sensory and intellectual functioning was also present when older adults with large losses in hearing and vision were excluded from the analysis (Baltes \& Lindenberger, 1995; Lindenberger \& Baltes, 1994). A third reason is based on the present finding that the high degree of predictive overlap between sensory functioning and age is also observed at younger ages when sensory deprivation is unlikely to be present, but only in that category of intellectual functioning - the fluid mechanics-in which individual differences, according to life span theory (Baltes, 1993; Horn \& Hofer, 1992), are dominated by factors associated with the current functional status of the brain.

As is often true for third-variable explanations, the common cause hypothesis in its current form is both theoretically and empirically underidentified. Some of the work needed to test its implications are under way in our laboratory. For instance, we are exploring whether temporary reductions in sensory performance levels through vision-impairing lenses and hearing-impairing earshields are able to simulate cognitive aging losses in middle-aged adults. According to the common cause hypothesis, simulated reductions of peripheral input should not result in major and generalized performance decrements in middle-aged adults because such treatments do not alter the neurophysiological status of the brain. In addition, we are in the process of 
examining whether sensory and sensorimotor tasks require an increasing amount of cognitive resources (e.g., attention) with advancing age. Finally, we are exploring the sensory demand characteristics of the cognitive tests of our battery. Except for some surplus covariance between visual acuity and perceptual speed, the evidence with older participants thus far does not suggest that cognitive tests with relatively high sensory demands or sensory specificity exhibit a stronger or more modality-specific relation to sensory functioning than tests with low or less specific sensory demands (see also Lindenberger \& Baltes, 1994, Table 7).

In conclusion, the present data suggest that a large portion of the mechanisms that drive negative age differences in sensory performance also bring about the aging of complex cognition. This finding has implications regarding the search for "psychological primitives" of negative age differences in cognition (Hertzog, 1996; Lindenberger \& Baltes, 1994; Salthouse, 1991a, $1991 \mathrm{~b}$ ) and gives new impetus to the dedifferentiation or neointegration hypothesis of adult intellectual development (Baltes, Cornelius, Spiro, Nesselroade, \& Willis, 1980; Reinert, 1970; Schaie, Willis, Jay, \& Chipuer, 1989). The very high degree of commonality between the age-related variance of the two domains is consistent with the notion that at least a major portion of these primitives is operating at a relatively global, rather than modular or domain-specific, level. At the same time, it is important to note that there also was some evidence for specificity in our data. For instance, in their role as predictors of individual differences in intelligence, hearing and age had more variance in common than vision and age, which is reminiscent of earlier findings regarding the diagnosticity of hearing loss for cognitive aging phenomena (Granick et al., 1976).

We argue, then, that the present findings open a new window to the investigation of negative age differences in adult cognition by redirecting the explanatory search. The strong connection between sensory and intellectual functioning in old age points to inquiries into sources, factors, and mechanisms that are common to both domains. Specifically, and in line with some earlier work (MacFarland, 1968; Walsh, 1976), our data suggest that the investigation of negative age differences in sensory and perceptual tasks may contribute in important ways to our understanding of aging losses in cognitive functioning. Most likely, sensory tasks are more easily amenable to task decomposition, cognitive psychophysics (e.g., Kliegl, Mayr, \& Krampe, 1994), and neuroscience procedures than complex cognitive tasks. Agecomparative research on sensory tasks using these and other approaches may enhance our understanding of negative age differences in adult cognitive development.

\section{References}

American Psychiatric Association. (1987). Diagnostic and statistical manual of mental disorders (3rd ed., rev.). Washington, DC: Author.

Anstey, K. J., Lord, S. R., \& Williams, P. (1997). Strength in the lower limbs, visual contrast sensitivity, and simple reaction time predict cognition in older women. Psychology and Aging, 12, 137-144.

Baltes, P. B. (1968). Longitudinal and cross-sectional sequences in the study of age and generation effects. Human Development, 11, 145171.

Baltes, P. B. ( 1987). Theoretical propositions of life-span developmental psychology: On the dynamics between growth and decline. Developmental Psychology, 23, 611-626.

Baltes, P. B. (1993). The aging mind: Potential and limits. The Gerontologist, 33, 580-594.

Baltes, P. B., Cornelius, S. W., Spiro, A., Nesselroade, J. R., \& Willis, S. L. (1980). Integration versus differentiation of fluid/crystallized intelligence in old age. Developmental Psychology, 16, 625-635.

Baltes, P. B., \& Lindenberger, U. (1995). Sensorik und Intelligenz: Intersysternische Wechselwirkungen und Veränderungen im hohen Alter [Sensory functioning and intelligence: Intersystemic dependencies and changes in old age]. Akademie-Journal, 1, 20-28.

Baltes, P. B., Mayer, K. U., Helmchen, H., \& Steinhagen-Thiessen, E. (1993). The Berlin Aging Study (BASE): Overview and design. Ageing and Society, 13, 483-515.

Bentler, P. M. (1989). EQS: Structural equations manual. Los Angeles: BMDP Statistical Software.

Carroll, J. B. (1993). Human cognitive abilities. Cambridge, England: Cambridge University Press.

Corso, J. F. (1987). Sensory-perceptual processes and aging. In K. W. Schaie \& C. Eisdorfer (Eds.), Annual review of gerontology and geriatrics (pp. 29-55). New York: Springer.

Craik, F. I. M., \& Salthouse, T. A. (Eds.). (1992). The handbook of aging and cognition. Hillsdale, NJ: Erlbaum.

Dixon, R. A., \& Baltes, P. B. (1986). Toward life-span research on the functions and pragmatics of intelligence. In R. J. Sternberg \& R. K. Wagner (Eds.), Practical intelligence: Nature and origins of competence in the everyday world (pp. 203-234). New York: Cambridge University Press.

Fozard, J. L. (1990). Vision and hearing in aging. In J. E. Birren \& K. W. Schaie (Eds.), Handbook of the psychology of aging (3rd ed., pp. 150-170). San Diego, CA: Academic Press.

Geigy, J. R. (1977). Wissenschaftliche Tabellen [Scientific tables]. Basel, Switzerland: J. R. Geigy AG.

Granick, S., Kleban, M. H., \& Weiss, A. D. (1976), Relationships between hearing loss and cognition in normally hearing aged persons. Journal of Gerontology, 31, 434-440.

Hermstein, R. J., \& Boring, E. G. (1965). A source book in the history of psychology. Cambridge, MA: Harvard University Press.

Hertzog, C. (1989). Influences of cognitive slowing on age differences in intelligence. Developmental Psychology, 25, 636-651.

Hertzog, C. (1990). On the utility of structural equation models for developmental research. In P. B. Baltes, D. L. Featherman, \& R. M. Lerner (Eds.), Life-span development and behavior (Vol. 10, pp. 257 290). Hillsdale, NJ: Erlbaum.

Hertzog, C. (1996). Research design in studies of aging and cognition. In J. E. Birren \& K. W. Schaie (Eds.), Handbook of the psychology of aging (4th ed.). New York: Academic Press.

Hilgard, E. R. (1987). Psychology in America: A historical survey. San Diego, CA: Harcourt Brace Jovanovich.

Horn, J. L. (1982). The theory of fluid and crystallized intelligence in relation to concepts of cognitive psychology and aging in adulthood. In F. I. M. Craik \& S. Trehub (Eds.), Aging and cognitive processes (pp. 237-278). New York: Plenum Press.

Horn, J. L., \& Hofer, S. M. (1992). Major abilities and development in the adult period. In R. J. Sternberg \& C. A. Berg (Eds.), Intellectual development (pp. 44-99). Cambridge, England: Cambridge University Press.

Hultsch, D. F., Hertzog, C., Small, B. J., McDonald-Miszczak, L., et al. (1992). Short-term longitudinal change in cognitive performance in later life. Psychology and Aging, 7, 571-584.

Kliegl, R., Mayr, U., \& Krampe, R. T. (1994). Time-accuracy functions for determining process and person differences: An application to cognitive aging. Cognitive Psychology, 26, 134-164.

Lindenberger, U., \& Baltes, P. B. (1994). Sensory functioning and intel- 
ligence in old age: A strong connection. Psychology and Aging, 9, 339-355.

Lindenberger, U., Mayr, U., \& Kliegl, R. (1993). Speed and intelligence in old age. Psychology and Aging, 8, 207-220.

MacFarland, R. A. (1968). The sensory and perceptual processes in aging. In K. W. Schaie (Ed.), Theory and methods of research on aging (pp. 9-52), Morgantown: West Virginia University.

Magnusson, D., Bergman, L. R., Rudinger, G., \& Törestad, B. (Eds.). (1991). Problems and methods in longitudinal research: Stability and change. Cambridge, England: Cambridge University Press.

McArdle, J. J., \& Nesselroade, J. R. (1994). Using multivariate data to structure developmental change. In S. H. Cohen \& H. W. Reese (Eds.), Life-span developmental psychology: Methodological contributions (pp. 223-267). Hillsdale, NJ: Erlbaum.

Meng, X.-L., Rosenthal, R., \& Rubin, D. B. (1992). Comparing correlated correlation coefficients. Psychological Bulletin, 111, 172-175.

Nesselroade, J. R., \& Baltes, P. B. (Eds.). (1979). Longitudinal research in the study of behavior and development. New York: Academic Press.

Nettelbeck, T., \& Rabbitt, P. (1992). Aging, cognitive performance, and mental speed. Intelligence, 16, 189-205.

Reinert, G. (1970). Comparative factor analytic studies of intelligence throughout the life span. In L. R. Goulet \& P. B. Baltes (Eds.), Lifespan developmental psychology: Research and theory (pp. 476-484). New York: Academic Press.

Salthouse, T. A. (1991a). Mediation of adult age differences in cognition by reductions in working memory and speed of processing. Psychological Science, 2, 179-183.

Salthouse, T. A. (1991b). Theoretical perspectives on cognitive aging. Hillsdale, NJ: Erlbaum.
Schaie, K. W. (1965). A general model for the study of developmental problems. Psychological Bulletin, 64, 92-107.

Schaie, K. W. (1995). Adult intellectual development: The Seattle Longitudinal Study. New York: Cambridge University Press.

Schaie, K. W., Baltes, P. B., \& Strother, C. R. (1964). A study of auditory sensitivity in advanced age. Journal of Gerontology. 19, 453457.

Schaie, K. W., Willis, S. L., Jay, G., \& Chipuer, H. (1989). Structural invariance of cognitive abilities across the adult life span: A crosssectional study. Developmental Psychology, 25, 652-662.

Steinhagen-Thiessen, E., \& Borchelt, M. (1993). Health differences in advanced old age. Ageing and Society, 13, 619-656.

Stelmach, G. E., \& Hömberg, V. (1993). (Eds.). Sensorimotor impairment in the elderly. Dordrecht, The Netherlands: Kluwer.

Sternberg, R. J., \& Detterman, D. K. (Eds.). (1986). What is intelligence? Norwood, $\mathrm{NJ}$ : Ablex.

Vernon, P. A. (1987). Speed of information-pracessing and intelligence. Norwood, NJ: Ablex.

Walsh, D. A. (1976). Age differences in central perceptual processing: A dichoptic backward masking investigation. Journal of Gerontology, $31,178-185$.

Welford, A. T. (1984). Between bodily changes and performance: Some possible reasons for slowing with age. Experimental Aging Research, $10,73-88$.

Received March 12, 1996

Revision received May 15, 1996

Accepted June 15, 1996 